\title{
Assessing the performance of density functional theory in optimizing molecular crystal structure parameters
}

Jack Binns, M ary Healy, Simon Parsons and Carole A. M orrison*

The School of Chemistry, University of Edinburgh, The King's Buildings, West M ains Road, Edinburgh, EH9 3JJ, UK.

\section{Supplementary Information}

Compound details and computational parameters applied to Class I and Class II data sets

Table S1 Class I (hydrogen bond interaction) compound identification and computational parameter setup

\begin{tabular}{|c|c|c|c|c|c|}
\hline CSD Reference & Compound name & $\begin{array}{l}\text { Unit cell dimensions } \\
\left(r / \AA, \angle /^{\circ}\right)\end{array}$ & $\begin{array}{l}\text { Plane-wave } \\
\text { basis set } \\
\text { energy cut- } \\
\text { off } \\
\left(\mathrm{E}_{\mathrm{cut})}\right.\end{array}$ & $\begin{array}{l}\text { Monkhurst- } \\
\text { Pack grid } \\
\text { (k-point } \\
\text { settings) }\end{array}$ & $\begin{array}{l}\text { No. k-points, } \\
\text { and actual k- } \\
\text { point spacing } \\
\left(\AA^{-1}\right)\end{array}$ \\
\hline ACEM ID03 & Acetamide & $\begin{array}{l}a=b=c=7.914 \\
\alpha=\beta=\gamma=93.3415\end{array}$ & 660 & $2 \times 2 \times 2$ & $\begin{array}{l}2 \text { k-points } \\
0.063412 \\
\times 0.063412 \\
\times 0.063412 \\
\end{array}$ \\
\hline ALOXAN11 & 2,4,5,6-pyrimidinetetrone & $\begin{array}{l}a=b=5.481 \\
c=13.853 \\
\alpha=\beta=\gamma=90\end{array}$ & 520 & $3 \times 3 \times 2$ & $\begin{array}{l}3 \text { k-points } \\
0.057068 \\
\times 0.057068 \\
\times 0.036093\end{array}$ \\
\hline BZAM ID02 & Benzamide & $\begin{array}{l}a=5.529, \\
b=5.033 \\
c=21.343 \\
\alpha=\gamma=90.0, \beta=88.73 .\end{array}$ & 600 & $3 \times 3 \times 1$ & $\begin{array}{l}4 \text { k-points } \\
0.060303 \\
\times 0.06623 \\
\times 0.046865\end{array}$ \\
\hline CBOHAZO2 & 1,3-diaminourea & $\begin{array}{l}a=3.618 \\
b=8.789 \\
c=12.487 \\
\alpha=\gamma=90.0, \beta=106.43\end{array}$ & 620 & $4 \times 2 \times 2$ & $\begin{array}{l}4 \text { k-points } \\
0.072041 \\
\times 0.056889 \\
\times 0.041746\end{array}$ \\
\hline FACETA01 & Monofluoracetamide & $\begin{array}{l}a=5.0974, \\
b=5.1531, \\
c=6.6501, \\
\alpha=102.52, \beta=101.33, \\
\gamma=99.54\end{array}$ & 620 & $3 \times 3 \times 2$ & $\begin{array}{l}9 \text { k-points } \\
0.06833 \\
\times 0.067889 \\
\times 0.079365\end{array}$ \\
\hline FORAM 001 & $\begin{array}{l}\text { N-hydroxy- } \\
\text { methaneimidamide }\end{array}$ & $\begin{array}{l}a=8.187 \\
b=7.111 \\
c=4.7609 \\
\alpha=\beta=\gamma=90.0\end{array}$ & 600 & $2 \times 2 \times 3$ & $\begin{array}{l}2 \text { k-points } \\
0.061072 \\
\times 0.070314 \\
\times 0.070015\end{array}$ \\
\hline GLOXIM 11 & Glyoxime & $\begin{array}{l}a=3.745, \\
b=4.398 \\
c=10.852, \\
\alpha=\gamma=90.0, \beta=92.41 .\end{array}$ & 620 & $4 \times 3 \times 2$ & $\begin{array}{l}8 \text { k-points } \\
0.066815 \\
\times 0.075792 \\
\times 0.046115\end{array}$ \\
\hline GUSRIV & $\begin{array}{l}\text { 2-(3-dimethylamino-1- } \\
\text { oxoprop-2-enyl)phenol }\end{array}$ & $\begin{array}{l}a=8.881 \\
b=10.100 \\
c=11.435\end{array}$ & 620 & $2 \times 2 \times 2$ & $\begin{array}{l}2 \text { k-points } \\
0.059798 \\
\times 0.049506 \\
\end{array}$ \\
\hline
\end{tabular}




\begin{tabular}{|c|c|c|c|c|c|}
\hline & & $\alpha=\gamma=90.0, \beta=109.69$ & & & $\times 0.046439$ \\
\hline JUQSUJ01 & Methyl $\alpha$-D-xylofuranoside & $\begin{array}{l}a=6.2238 \\
b=8.1389 \\
c=7.3637 \\
\alpha=\gamma=90.0, \beta=101.15\end{array}$ & 620 & $3 \times 2 \times 2$ & $\begin{array}{l}3 \text { k-points } \\
0.054588 \\
\times 0.061433 \\
\times 0.069206\end{array}$ \\
\hline M ALNAC07 & Perdeuteromalonic acid & $\begin{array}{l}a=10.663, \\
b=5.142, \\
c=11.234, \\
\alpha=103.39, \beta=136.81, \\
\gamma=85.19 .\end{array}$ & 680 & $2 \times 3 \times 2$ & $\begin{array}{l}6 \text { k-points } \\
0.069077 \\
\times 0.067187 \\
\times 0.067161\end{array}$ \\
\hline M ELAM I06 & 1,3,-triazine-2,4,6-triamine & $\begin{array}{l}a=10.4330 \\
b=7.4580 \\
c=7.2380 \\
\alpha=\gamma=90.0, \beta=113.30\end{array}$ & 480 & $2 \times 2 \times 2$ & $\begin{array}{l}2 \text { k-points } \\
0.05218 \\
\times 0.067042 \\
\times 0.075214\end{array}$ \\
\hline NALCYSO2 & N-aceyl-L-cysteine & $\begin{array}{l}a=5.766 \\
b=6.433 \\
c=5.014 \\
\alpha=102.8, \beta=102.77, \\
\gamma=95.81\end{array}$ & 600 & $3 \times 3 \times 3$ & $\begin{array}{l}14 \text { k-points } \\
0.06003 \\
\times 0.053812 \\
\times 0.070429\end{array}$ \\
\hline RIWQET01 & $\begin{array}{l}\text { 2-(methylbenzylamino)-3,5- } \\
\text { dinitropyridine }\end{array}$ & $\begin{array}{l}a=8.352 \\
b=8.570 \\
c=8.909 \\
\alpha=\gamma=90.0, \beta=93.98\end{array}$ & 460 & $2 \times 2 \times 2$ & $\begin{array}{l}2 \text { k-points } \\
0.060011 \\
\times 0.058343 \\
\times 0.056259\end{array}$ \\
\hline SUBRAC01 & Octane1,8-dioic acid & $\begin{array}{l}a=8.710 \\
b=5.0885 \\
c=9.815 \\
\alpha=\gamma=90.0, \beta=95.07 .\end{array}$ & 620 & $2 \times 3 \times 2$ & $\begin{array}{l}4 \text { k-points } \\
0.057631 \\
\times 0.065507 \\
\times 0.051143\end{array}$ \\
\hline TACETA09 & $\begin{array}{l}\text { 3,4-diacetylhexa-2,4-diene- } \\
\text { 2,5-diol }\end{array}$ & $\begin{array}{l}a=9.0859 \\
b=9.2413 \\
c=11.465 \\
\alpha=\beta=\gamma=90.0\end{array}$ & 600 & $2 \times 2 \times 2$ & $\begin{array}{l}1 \mathrm{k}-\text { point } \\
0.05503 \\
\times 0.054105 \\
\times 0.043611\end{array}$ \\
\hline TRAZOL02 & 1,2,4-triazole & $\begin{array}{l}a=9.748 \\
b=9.331 \\
c=6.927 \\
\alpha=\beta=\gamma=90.0\end{array}$ & 420 & $2 \times 2 \times 2$ & $\begin{array}{l}1 \mathrm{k}-\text { point } \\
0.051293 \\
\times 0.053585 \\
\times 0.072181\end{array}$ \\
\hline UREAXX12 & urea & $\begin{array}{l}a=b=5.565 \\
c=4.684 \\
\alpha=\beta=\gamma=90.0\end{array}$ & 520 & $3 \times 3 \times 3$ & $\begin{array}{l}6 \text { k-points } \\
0.059898 \\
\times 0.059898 \\
\times 0.071164\end{array}$ \\
\hline
\end{tabular}


Table S2 Class II (dispersion interaction) compound identification and computational parameter setup

\begin{tabular}{|c|c|c|c|c|c|}
\hline CSD Reference & Compound name & $\begin{array}{l}\text { Unit cell } \\
\text { dimensions }\end{array}$ & $\begin{array}{l}\text { Plane- } \\
\text { wave } \\
\text { basis set } \\
\text { energy } \\
\text { cut-off } \\
\left(\mathrm{E}_{\text {cut }}\right)\end{array}$ & $\begin{array}{l}\text { Monkhurst- } \\
\text { Pack grid } \\
\text { (k-point } \\
\text { settings) }\end{array}$ & $\begin{array}{l}\text { No. k-points, and } \\
\text { actual k-point } \\
\text { spacing }\left(\AA^{-1}\right)\end{array}$ \\
\hline ACETYL05 & Dideutero-acetylene & $\begin{array}{l}a=6.19 \\
b=6.00 \\
c=5.546 \\
a=\beta=?=90.0\end{array}$ & 460 & $2 \times 3 \times 2$ & $\begin{array}{l}2 \text { k-points } \\
0.08082 \times 0.055546 \\
\times 0.090155\end{array}$ \\
\hline GIPVUW02 & $\begin{array}{l}\text { 2,5-Diaza-1,6-dioxa-6a- } \\
\text { thiapentalene }\end{array}$ & $\begin{array}{l}a=6.76 \\
b=6.92 \\
c=10.90 \\
a=\beta=90.0 \\
?=125.40\end{array}$ & 640 & $2 \times 2 \times 2$ & $\begin{array}{l}2 \text { k-points } \\
0.079846 \\
\times 0.072202 \\
\times 0.04952\end{array}$ \\
\hline ANTCEN16 & Decadeutero-anthracene & $\begin{array}{l}a=8.37 \\
b=6.00 \\
c=11.12 \\
a=?=90.0 \\
\beta=125.40\end{array}$ & 500 & $2 \times 2 \times 2$ & $\begin{array}{l}2 \text { k-points } \\
0.073286 \\
\times 0.083333 \\
\times 0.055162\end{array}$ \\
\hline HXMTAM10 & Hexamethylenetetramine & $\begin{array}{l}a=b=c=5.9993 \\
a=\beta=?=109.471\end{array}$ & 420 & $3 \times 3 \times 3$ & $\begin{array}{l}4 \text { k-points } \\
0.068049 \\
\times 0.068049 \\
\times 0.068049\end{array}$ \\
\hline NBONAN01 & $\begin{array}{l}\text { exo,exo-2,3-Norborn-5- } \\
\text { enedicarboxylic anhydride }\end{array}$ & $\begin{array}{l}a=7.362 \\
b=7.968 \\
c=12.500 \\
a=\beta=?=90.0\end{array}$ & 620 & $2 \times 2 \times 1$ & $\begin{array}{l}1 \mathrm{k} \text {-point } \\
0.067916 \\
\times 0.062751 \times 0.08\end{array}$ \\
\hline NURWOM03 & 2,6-Dimethylpyrazine & $\begin{array}{l}a=7.287 \\
b=10.725 \\
c=7.452 \\
a=?=90.0 \\
\beta=90.37\end{array}$ & 500 & $2 \times 2 \times 2$ & $\begin{array}{l}2 \text { k-points } \\
0.068617 \times 0.04662 \\
\times 0.067097\end{array}$ \\
\hline BENZEN06 & Hexadeutero-benzene & $\begin{array}{l}a=7.360 \\
b=9.375 \\
c=6.703 \\
a=\beta=?=90.0\end{array}$ & 480 & $2 \times 2 \times 2$ & $\begin{array}{l}2 \text { k-points } \\
0.078406 \\
\times 0.078406 \\
\times 0.069979\end{array}$ \\
\hline OCHTET13* & $\begin{array}{l}\text { 1,3,5,7-tetranitro-1,3,5,7 } \\
\text {-tetraazacyclooctane }\end{array}$ & $\begin{array}{l}a=6.521, \\
b=10.761, \\
c=7.306 \\
a=?=90.0, \\
\beta=102.06 .\end{array}$ & 640 & $2 \times 2 \times 2$ & $\begin{array}{l}2 \text { k-points } \\
0.078406 \\
\times 0.046464 \\
\times 0.069979\end{array}$ \\
\hline NAPHTA14* & Perdeuteronaphthalene & $\begin{array}{l}a=8.096 \\
b=5.941 \\
c=8.648 \\
a=?=90.0 \\
\beta=124.63\end{array}$ & 500 & $2 \times 2 \times 2$ & $\begin{array}{l}2 \text { k-points } \\
0.075056 \\
\times 0.084161 \\
\times 0.070265\end{array}$ \\
\hline POCTUW & Trichlorofluoromethane & $\begin{array}{l}a=15.511 \\
b=5.879 \\
c=9.526 \\
a=\beta=?=90.0\end{array}$ & 620 & $1 \times 2 \times 2$ & $\begin{array}{l}1 \text { k-points } \\
0.06447 \times 0.085051 \\
\times 0.052487\end{array}$ \\
\hline TFMETH03* & Tetrafluoromethane & $\begin{array}{l}a=b=4.73845 \\
c=8.4800 \\
a=\beta=117.027\end{array}$ & 640 & $4 \times 4 \times 2$ & $\begin{array}{l}10 \text { k-points } \\
0.067358 \\
\times 0.067358 \\
\end{array}$ \\
\hline
\end{tabular}




\begin{tabular}{|l|l|l|l|l|l|}
\hline & & $?=54.2387$ & & & $\times 0.068573$ \\
\hline ZZZUEE04* & Strychnine & $a=11.326$, & 580 & $1 \times 1 \times 1$ & $1 \mathrm{k}-$ point \\
& & $b=11.765$, & 0.084993 \\
& & $c=11.994$, & & $\times 0.12081 \times 0.083625$ \\
& & $a=\beta=?=90.0$. & & \\
\hline BIPHEN08 & Decadeutero-biphenyl & $a=7.780$, & 500 & $2 \times 2 \times 2$ & $2 \mathrm{k}-$ points \\
& & $b=5.565$, & & 0.064417 \\
& & $c=9.440$, & & $\times 0.089847$ \\
& & $a=?=90.0$, & & & \\
& & $\beta=93.90$. & & & \\
\end{tabular}

* X-ray data collection. 
Figure S1 Convergence graphs with respect to (a) Plane wave basis set energy cut-off and (b) M onkhurst Pack k-point setting grids for model system NURWOM 3 (2,6-Dimethylpyrazine)

(a)
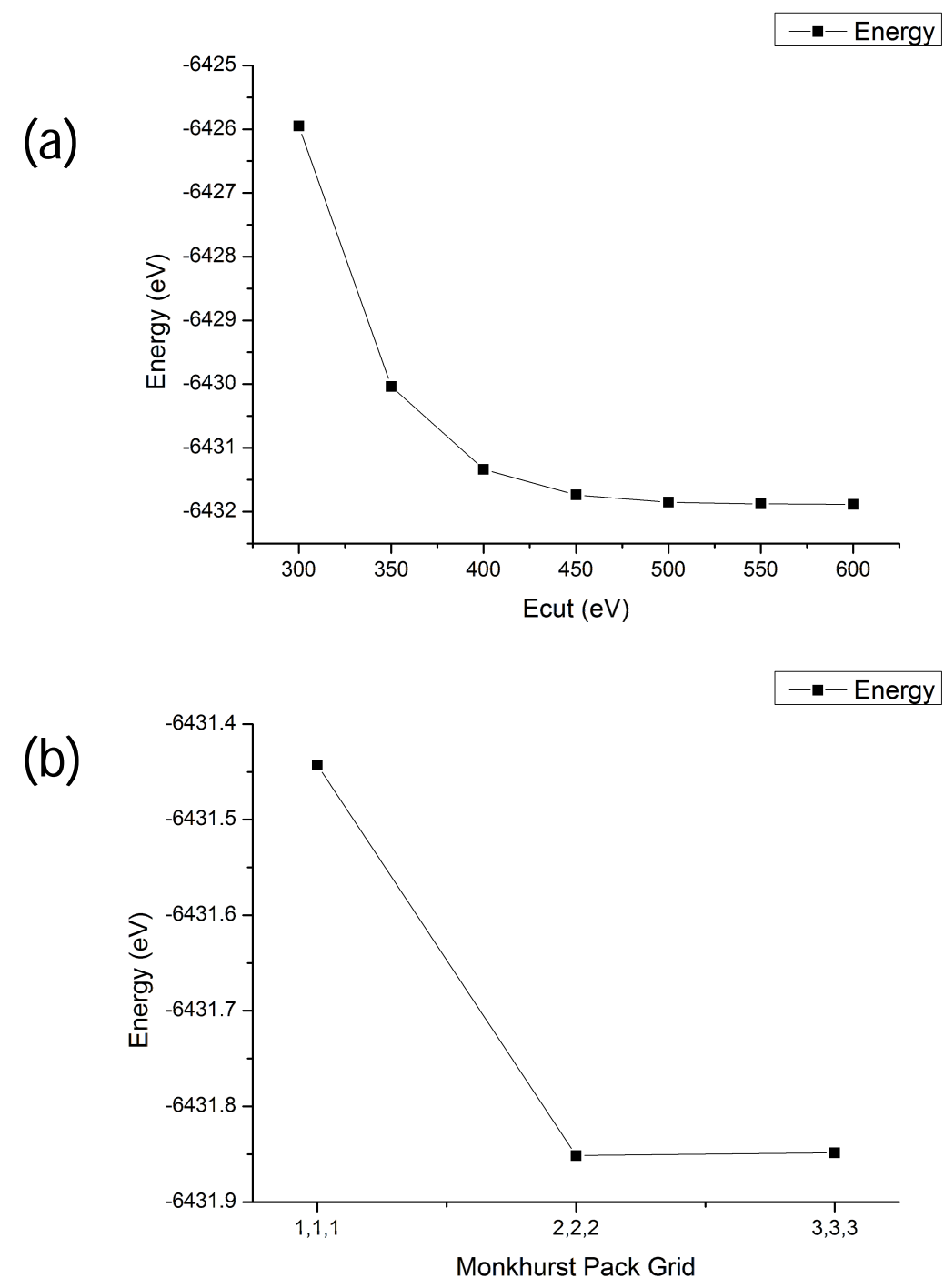
Table S3 Class I calculated crystal packing similarity (CPS) scores

\begin{tabular}{|c|c|c|c|c|c|c|c|c|c|c|c|c|}
\hline \multirow[b]{2}{*}{ CSD Reference } & \multicolumn{2}{|c|}{ PBE } & \multicolumn{2}{|c|}{ PW91 } & \multicolumn{2}{|c|}{ PBEsol } & \multicolumn{2}{|c|}{ PBE-TS } & \multicolumn{2}{|c|}{ PW91-OBS } & \multicolumn{2}{|c|}{ PBE-G06 } \\
\hline & $\begin{array}{l}\text { Fix } \\
\text { cell }\end{array}$ & $\begin{array}{l}\text { Opt } \\
\text { cell }\end{array}$ & $\begin{array}{l}\text { Fix } \\
\text { cell }\end{array}$ & $\begin{array}{l}\text { Opt } \\
\text { cell }\end{array}$ & $\begin{array}{l}\text { Fix } \\
\text { cell }\end{array}$ & $\begin{array}{l}\text { Opt } \\
\text { cell }\end{array}$ & $\begin{array}{l}\text { Fix } \\
\text { cell }\end{array}$ & $\begin{array}{l}\text { Opt } \\
\text { cell }\end{array}$ & $\begin{array}{l}\text { Fix } \\
\text { cell }\end{array}$ & $\begin{array}{l}\text { Opt } \\
\text { cell }\end{array}$ & $\begin{array}{l}\text { Fix } \\
\text { cell }\end{array}$ & $\begin{array}{l}\text { Opt } \\
\text { cell }\end{array}$ \\
\hline ACEM ID03 & 0.008 & 0.078 & 0.008 & 0.071 & 0.122 & 0.039 & 0.008 & 0.02 & 0.009 & 0.223 & 0.008 & 0.065 \\
\hline ALOXAN11 & 0.009 & 0.319 & 0.009 & 0.384 & 0.007 & 0.099 & 0.009 & 0.091 & 0.009 & 0.052 & 0.011 & 0.047 \\
\hline BZAMID02 & 0.005 & 0.671 & 0.005 & 0.732 & 0.056 & 0.298 & 0.005 & 0.027 & 0.053 & 0.465 & 0.007 & 0.15 \\
\hline CBOHAZO2 & 0.02 & 0.288 & 0.022 & 0.35 & 0.032 & 0.118 & 0.021 & 0.047 & 0.072 & 0.252 & 0.022 & 0.083 \\
\hline FACETA01 & 0.013 & 0.405 & 0.013 & 0.463 & 0.056 & 0.183 & 0.014 & 0.06 & 0.038 & 0.226 & 0.015 & 0.09 \\
\hline FORAM 001 & 0.038 & 0.411 & 0.041 & 0.422 & 0.11 & 0.257 & 0.035 & 0.112 & 0.089 & 0.239 & 0.034 & 0.083 \\
\hline GLOXIM 11 & 0.056 & 0.469 & 0.057 & 0.572 & 0.086 & 0.204 & 0.051 & 0.108 & 0.056 & 0.135 & 0.054 & 0.09 \\
\hline GUSRIV & 0.011 & 0.574 & 0.011 & 0.618 & 0.018 & 0.117 & 0.011 & 0.015 & 0.098 & 0.446 & 0.011 & 0.131 \\
\hline JUQSUJ01 & 0.012 & 0.513 & 0.012 & 0.461 & 0.073 & 0.234 & 0.027 & 0.067 & 0.057 & 0.451 & 0.011 & 0.092 \\
\hline MALNAC07 & 0.047 & 0.475 & 0.044 & 0.474 & 0.093 & 0.262 & 0.045 & 0.081 & 0.05 & 0.117 & 0.038 & 0.053 \\
\hline MELAM I06 & 0.013 & 0.38 & 0.023 & 0.428 & 0.04 & 0.214 & 0.013 & 0.058 & 0.045 & 0.261 & 0.03 & 0.101 \\
\hline NALCYSO2 & 0.015 & 0.506 & 0.015 & 0.596 & 0.067 & 0.174 & 0.021 & 0.066 & 0.057 & 0.253 & 0.016 & 0.05 \\
\hline RIWQET01 & 0.01 & 0.922 & 0.01 & 0.781 & 0.016 & 0.653 & 0.014 & 0.121 & 0.108 & 0.3 & 0.01 & 0.012 \\
\hline SUBRAC01 & 0.014 & 0.746 & 0.02 & 0.878 & 0.045 & 0.308 & 0.021 & 0.057 & 0.195 & 0.691 & 0.011 & 0.167 \\
\hline TACETA09 & 0.007 & 0.483 & 0.159 & 0.667 & 0.022 & 0.111 & 0.006 & 0.3 & 0.007 & 0.48 & 0.005 & 0.114 \\
\hline TRAZOL02 & 0.007 & 0.601 & 0.062 & 0.679 & 0.11 & 0.294 & 0.007 & 0.043 & 0.008 & 0.13 & 0.008 & 0.054 \\
\hline UREAXX12 & 0.011 & 0.179 & 0.012 & 0.18 & 0.016 & 0.037 & 0.011 & 0.02 & 0.013 & 0.232 & 0.01 & 0.09 \\
\hline
\end{tabular}

Table S4 Class II calculated crystal packing similarity (CPS) scores

\begin{tabular}{|c|c|c|c|c|c|c|c|c|c|c|c|c|c|}
\hline \multirow[t]{2}{*}{ CSD Reference } & \multicolumn{2}{|c|}{ PBE } & \multicolumn{2}{|c|}{ PW91 } & \multicolumn{2}{|c|}{ PBEsol } & \multicolumn{2}{|c|}{ PBE-TS } & \multicolumn{2}{|c|}{ PW91-OBS } & \multicolumn{2}{|c|}{ PBE-G06 } & \multirow{2}{*}{$\begin{array}{l}\text { PBE- } \\
\text { M BD } \\
\text { Opt } \\
\text { cell }\end{array}$} \\
\hline & $\begin{array}{l}\text { Fix } \\
\text { cell }\end{array}$ & $\begin{array}{l}\text { Opt } \\
\text { cell }\end{array}$ & $\begin{array}{l}\text { Fix } \\
\text { cell }\end{array}$ & $\begin{array}{l}\text { Opt } \\
\text { cell }\end{array}$ & $\begin{array}{l}\text { Fix } \\
\text { cell }\end{array}$ & $\begin{array}{l}\text { Opt } \\
\text { cell }\end{array}$ & $\begin{array}{l}\text { Fix } \\
\text { cell }\end{array}$ & $\begin{array}{l}\text { Opt } \\
\text { cell }\end{array}$ & $\begin{array}{l}\text { Fix } \\
\text { cell }\end{array}$ & $\begin{array}{l}\text { Opt } \\
\text { cell }\end{array}$ & $\begin{array}{l}\text { Fix } \\
\text { cell }\end{array}$ & $\begin{array}{l}\text { Opt } \\
\text { cell }\end{array}$ & \\
\hline ACETYL05 & 0.045 & 0.277 & 0.044 & 0.321 & 0.045 & 0.13 & 0.045 & 0.092 & 0.044 & 0.428 & 0.045 & 0.207 & \\
\hline GIPVUW02 & 0.065 & 0.482 & 0.107 & 0.697 & 0.104 & 0.221 & 0.071 & 0.161 & 0.107 & 0.149 & 0.038 & 0.128 & 0.126 \\
\hline ANTCEN16 & 0.017 & 0.846 & 0.018 & 1.013 & 0.021 & 0.249 & 0.017 & 0.037 & 0.016 & 0.457 & 0.013 & 0.209 & \\
\hline HXM TAM 10 & 0.004 & 0.379 & 0.005 & 0.294 & 0.005 & 0.065 & 0.005 & 0.014 & 0.006 & 0.461 & 0.007 & 0.142 & \\
\hline NBONAN01 & 0.009 & 0.328 & 0.011 & 0.389 & 0.007 & 0.099 & 0.019 & 0.036 & 0.197 & 0.334 & 0.01 & 0.086 & \\
\hline NURWOM 03 & 0.006 & 0.56 & 0.007 & 0.713 & 0.006 & 0.121 & 0.006 & 0.094 & 0.012 & 0.579 & 0.007 & 0.179 & 0.061 \\
\hline BENZEN06 & 0.002 & 0.544 & 0.002 & 0.657 & 0.004 & 0.001 & 0.001 & 0.001 & 0.001 & 0.177 & 0.001 & 0.177 & 0.002 \\
\hline OCHTET13* & 0.045 & 0.478 & 0.014 & 0.53 & 0.053 & 0.18 & 0.051 & 0.18 & 0.038 & 0.111 & 0.035 & 0.091 & \\
\hline NAPHTA14* & 0.007 & 0.53 & 0.008 & 0.609 & 0.01 & 0.156 & 0.007 & 0.018 & 0.007 & 0.462 & 0.006 & 0.235 & \\
\hline POCTUW & 0.042 & 0.418 & 0.044 & 0.46 & 0.048 & 0.109 & 0.027 & 0.108 & 0.046 & 0.215 & 0.044 & 0.029 & \\
\hline TFM ETH03* & 0.012 & 0.452 & 0.012 & 0.448 & 0.008 & 0.408 & 0.012 & 0.11 & 0.0012 & 0.248 & 0.013 & 0.027 & \\
\hline ZZZUEE04* & 0.013 & 0.527 & 0.012 & 0.579 & 0.018 & 0.018 & 0.014 & 0.016 & 0.108 & 0.614 & 0.012 & 0.125 & \\
\hline BIPHEN08 & 0.016 & 0.76 & 0.016 & 0.96 & 0.018 & 0.187 & 0.016 & 0.033 & 0.015 & 0.511 & 0.014 & 0.226 & \\
\hline
\end{tabular}


Table S5 Class I calculated unit cell volume

\begin{tabular}{|l|r|r|r|r|r|r|r|}
\hline $\begin{array}{l}\text { CSD } \\
\text { reference }\end{array}$ & \multicolumn{1}{l|l}{ Exp } & \multicolumn{1}{l|}{ PBE } & \multicolumn{1}{l|}{ PBEs0l } & \multicolumn{1}{l|}{ PBE-TS } & \multicolumn{1}{l|}{ PW1-OBS } & \multicolumn{1}{l|}{ PBE-G06 } \\
\hline ACEM ID03 & 492.95 & 514.77 & 513.12 & 482.98 & 487.40 & 432.87 & 474.60 \\
\hline ALOXAN11 & 472.63 & 556.31 & 574.05 & 489.47 & 495.93 & 485.43 & 484.11 \\
\hline BZAM ID02 & 593.78 & 751.62 & 778.63 & 644.40 & 587.87 & 489.96 & 557.74 \\
\hline CBOHAZ02 & 380.86 & 429.26 & 439.19 & 374.41 & 379.86 & 332.40 & 367.31 \\
\hline CYCACHZ01 & 440.93 & 512.61 & 515.06 & 513.30 & 432.77 & 403.45 & 432.77 \\
\hline FACETA01 & 163.20 & 198.23 & 202.52 & 172.03 & 166.43 & 146.18 & 156.92 \\
\hline FORAM 001 & 277.17 & 319.00 & 320.60 & 282.11 & 277.21 & 241.37 & 264.94 \\
\hline GLOXIM 11 & 178.58 & 219.65 & 224.56 & 182.81 & 179.88 & 165.30 & 171.72 \\
\hline GUSRIV & 965.72 & 1196.91 & 1236.93 & 1007.56 & 964.40 & 803.41 & 921.26 \\
\hline JUQSUJ01 & 365.97 & 427.58 & 435.36 & 368.84 & 360.48 & 301.35 & 350.63 \\
\hline MALNAC07 & 406.75 & 508.84 & 531.44 & 444.36 & 414.96 & 388.70 & 404.88 \\
\hline MELAM 106 & 517.25 & 614.97 & 621.89 & 533.17 & 511.85 & 466.21 & 493.30 \\
\hline NALCYS02 & 174.66 & 212.58 & 218.84 & 182.78 & 178.86 & 157.71 & 171.78 \\
\hline RIWQET01 & 636.14 & 966.94 & 924.36 & 772.31 & 659.94 & 587.12 & 637.03 \\
\hline SUBRAC01 & 433.31 & 551.57 & 573.75 & 474.10 & 428.98 & 344.90 & 410.09 \\
\hline TACETA09 & 962.66 & 1173.92 & 798.97 & 1006.44 & 962.40 & 798.95 & 919.03 \\
\hline TRAZOL02 & 630.07 & 809.13 & 842.43 & 702.69 & 626.24 & 581.45 & 611.73 \\
\hline UREAXX12 & 145.06 & 158.71 & 158.56 & 142.64 & 146.52 & 128.13 & 138.79 \\
\hline
\end{tabular}

Table S6 Class II calculated unit cell volume

\begin{tabular}{|l|r|r|r|r|r|r|r|l|}
\hline Compound name & Exp & PBE & PW91 & PBEsol & PBE-TS & \multicolumn{1}{l|}{$\begin{array}{l}\text { PW1- } \\
\text { OBS }\end{array}$} & $\begin{array}{l}\text { PBE- } \\
\text { G06 }\end{array}$ & $\begin{array}{l}\text { PBE- } \\
\text { M BD }\end{array}$ \\
\hline ACETYL05 & 102.97 & 116.70 & 122.16 & 98.38 & 97.46 & 81.94 & 91.17 & -- \\
\hline GIPVUW02 & 472.81 & 602.23 & 644.53 & 521.76 & 501.32 & 497.82 & 483.38 & 501.07 \\
\hline ANTCEN16 & 455.21 & 589.18 & 618.08 & 489.67 & 450.81 & 379.83 & 417.31 & -- \\
\hline HXM TAM 0 & 166.22 & 198.65 & 191.07 & 171.50 & 167.32 & 131.79 & 155.01 & -- \\
\hline NBONAN01 & 733.26 & 848.87 & 872.33 & 766.46 & 733.21 & 638.82 & 705.77 & -- \\
\hline NURWOM 03 & 582.38 & 726.27 & 766.00 & 606.35 & 569.12 & 455.85 & 539.99 & 567.41 \\
\hline BENZEN06 & 462.51 & 611.90 & 646.53 & 464.53 & 462.59 & 419.49 & 419.49 & 462.45 \\
\hline OCHTET13 & 501.37 & 599.88 & 610.74 & 538.08 & 530.35 & 512.54 & 513.15 & -- \\
\hline NAPTHA14 & 342.26 & 428.65 & 442.14 & 360.64 & 339.73 & 280.06 & 312.43 & -- \\
\hline POCTUW & 868.65 & 1068.84 & 1094.13 & 908.66 & 919.81 & 974.57 & 865.51 & -- \\
\hline TFMETH03 & 132.85 & 175.80 & 175.43 & 171.27 & 141.69 & 155.22 & 132.69 & -- \\
\hline ZZUEE04 & 1598.21 & 1912.46 & 1945.04 & 1602.34 & 1594.79 & 1311.79 & 1533.40 & -- \\
\hline BIPHEN08 & 407.76 & 523.29 & 555.47 & 435.36 & 403.34 & 334.03 & 375.83 & -- \\
\hline
\end{tabular}


Table S7 Class I Calculated unit cell shape (longest unit cell length/shortest unit cell length)

\begin{tabular}{|l|l|l|l|l|l|l|l|}
\hline $\begin{array}{l}\text { CSD } \\
\text { reference }\end{array}$ & Exp & PBE & PW91 & PBEsol & PBE-TS & PW1-OBS & PBE-G06 \\
\hline ACEM ID03 & N/A* & N/A & N/A & N/A & N/A & N/A & N/A \\
\hline ALOXAN11 & 2.37168 & 2.39099 & 2.2571 & 2.30719 & 2.36505 & 2.36842 & 2.36534 \\
\hline BZAM ID02 & 4.24061 & 4.60626 & 4.73255 & 4.44463 & 4.24788 & 4.17854 & 4.18371 \\
\hline CBOHAZ02 & 3.45135 & 3.11559 & 3.04153 & 3.30618 & 3.40327 & 3.55498 & 3.43368 \\
\hline CYCACHZ01 & 1.19746 & 1.03682 & 1.02763 & 1.03549 & 1.21263 & 1.22968 & 1.21263 \\
\hline FACETA01 & 1.30461 & 1.44519 & 1.47488 & 1.35321 & 1.32359 & 1.24933 & 1.28204 \\
\hline FORAM 001 & 1.71963 & 1.66774 & 1.65294 & 1.66673 & 1.73194 & 1.74956 & 1.74956 \\
\hline GLOXIM 11 & 2.89773 & 2.5002 & 2.42932 & 2.74596 & 2.83812 & 2.95354 & 2.91327 \\
\hline GUSRIV & 1.28767 & 1.36867 & 1.38381 & 1.30614 & 1.28654 & 1.24172 & 1.27943 \\
\hline JUQSUJ01 & 1.30771 & 1.33271 & 1.33717 & 1.3232 & 1.31828 & 1.36036 & 1.32021 \\
\hline MALNAC07 & 2.18475 & 2.03743 & 1.97872 & 2.06516 & 2.1675 & 2.21135 & 2.17622 \\
\hline MELAM 106 & 1.44142 & 1.52401 & 1.52538 & 1.48092 & 1.46506 & 1.4727 & 1.44087 \\
\hline NALCYS02 & 1.28301 & 1.2298 & 1.22039 & 1.26029 & 1.27852 & 1.27086 & 1.29305 \\
\hline RIWQET01 & 1.03956 & 1.11296 & 1.10652 & 1.07743 & 1.05061 & 1.07323 & 1.03903 \\
\hline SUBRAC01 & 1.92886 & 1.8774 & 1.86757 & 1.90031 & 1.93833 & 1.98062 & 1.93375 \\
\hline TACETA09 & 1.26185 & 1.26351 & 1.39322 & 1.25705 & 1.26255 & 1.39351 & 1.24822 \\
\hline TRAZOL02 & 1.40725 & 1.14369 & 1.10828 & 1.24477 & 1.38032 & 1.42842 & 1.4029 \\
\hline UREAXX12 & 1.18809 & 1.23019 & 1.23249 & 1.20054 & 1.1884 & 1.13511 & 1.16232 \\
\hline
\end{tabular}

* cubic system

Table S8 Class II Calculated unit cell shape (longest unit cell length/shortest unit cell length)

\begin{tabular}{|l|l|l|l|l|l|l|l|l|}
\hline Compound name & Exp & PBE & PW91 & PBEsol & PBE-TS & $\begin{array}{l}\text { PW1- } \\
\text { OBS }\end{array}$ & $\begin{array}{l}\text { PBE- } \\
\text { G06 }\end{array}$ & $\begin{array}{l}\text { PBE- } \\
\text { M BD }\end{array}$ \\
\hline ACETYL05 & 1.51457 & 1.41136 & 1.39018 & 1.43384 & 1.49479 & 1.53593 & 1.49921 & \\
\hline GIPVUW02 & 1.61239 & 1.50555 & 1.39738 & 1.56752 & 1.59515 & 1.59985 & 1.63769 & 1.56989 \\
\hline ANTCEN16 & 1.85333 & 1.79328 & 1.78265 & 1.83934 & 1.84945 & 1.86919 & 1.86973 & -- \\
\hline HXM TAM 0 & 1.69791 & 1.68118 & 1.68294 & 1.70683 & 1.69826 & 1.69752 & 1.70896 & -- \\
\hline NBONAN01 & 1.4718 & 1.36712 & 1.33663 & 1.43243 & 1.47304 & 1.61442 & 1.50553 & 1.46 \\
\hline NURWOM 03 & 1.39863 & 1.35887 & 1.35314 & 1.39897 & 1.39868 & 1.4146 & 1.4146 & 1.3986 \\
\hline BENZEN06 & 1.65023 & 1.72423 & 1.72605 & 1.68541 & 1.70585 & 1.69002 & 1.67073 & -- \\
\hline OCHTET13 & 1.45565 & 1.43221 & 1.43104 & 1.45371 & 1.45652 & 1.42499 & 1.46469 & -- \\
\hline NAPTHA14 & 2.63848 & 2.65302 & 2.6539 & 2.69468 & 2.63819 & 2.70609 & 2.63169 & -- \\
\hline POCTUW & 1.78961 & 1.78086 & 1.78052 & 1.78318 & 1.77666 & 1.77652 & 1.78191 & -- \\
\hline TFMETH03 & 1.05898 & 1.08093 & 1.08239 & 1.05841 & 1.05817 & 1.04522 & 1.04353 & -- \\
\hline ZZZUEE04 & 1.69632 & 1.65127 & 1.63731 & 1.68732 & 1.69994 & 1.67136 & 1.70974 & -- \\
\hline BIPHEN08 & 1.51457 & 1.41136 & 1.39018 & 1.43384 & 1.49479 & 1.53593 & 1.49921 & \\
\hline
\end{tabular}

